\title{
Perfil epidemiológico de adultos jovens (20 a 24 anos) com HIV/AIDS em uma cidade do interior paulista
}

\author{
Epidemiological profile of young adults (20 to 24 years) with HIV/AIDS from a town in the \\ hinterland of São Paulo state
}

Perfil epidemiológico de adultos jóvenes (20 a 24 años) con VIH/SIDA de una ciudad en el interior del estado de San Pablo

Igor Nascimento Alves ${ }^{1 *}$, Luís Antônio Soares Pires Filho ${ }^{1}$, Ana Carolina Soares Salviano ${ }^{1}$, Caio Alves Santos ${ }^{1}$, Gabriel Henrique Gastaldello ${ }^{1}$, Gabriel Nunes Pinheiro', Leonardo Delsin Magri ${ }^{1}$, Marcelo Vinícius Donley Wirgues ${ }^{1}$.

\section{RESUMO}

Objetivo: Analisar a incidência e descrever os coeficientes de adultos jovens, 20 a 24 anos, com HIV/AIDS, no período de 2007 a 2017. Métodos: Os dados de notificação foram retirados da base de dados do Sistema de Informações de Agravos de Notificação (SINAN), via Portal de Saúde do SUS (DataSUS). Estes foram tabulados em gráficos no programa Excel, sendo representadas as variáveis: padrões de incidência em cada ano e de maneira comparativa entre os anos, segundo sexo, faixa etária, escolaridade e cor/raça. Resultados: Foram registrados 1124 casos $(n=1124)$ de HIV/AIDS no período de 2007 a 2017 no município de Ribeirão Preto - SP. Na categoria de exposição homens que fazem sexo com homens (HSH), evidenciou de 603 casos $(n=603)$. Em relação a variável de gênero, a população estudada constitui-se em sua maioria de homens $70,37 \%(n=1102)$; raça/cor branca, 634 casos $(n=634)$. No que diz respeito à escolaridade, em homens, os resultados demonstraram que em 2007, 27,5\% ( $n=14)$ apresentavam ensino médio completo e em 2017, 56,2\% ( $n=90)$. Considerações Finais: Conclui-se que houve um aumento na incidência dos casos de HIV/AIDS em adultos, no período analisado.

Palavras-chave: Perfil de saúde, Adulto jovem, Síndrome da imunodeficiência adquirida.

\begin{abstract}
Objective: To determine the incidence and to report the coefficients of young adults with HIV/AIDS aged 20 to 24 years, in the period from 2007 to 2017. Methods: Notification data were obtained from the database of the System of Information about Notifiable Diseases (SINAN) via the Health Portal of SUS (DataSUS). The data were tabulated using Excel graphs, with representation of the following variables: patterns of incidence for each year and comparatively from year to year according to sex, age range, schooling, and color/race. Results: A total of 1124 cases ( $n=1124$ ) of HIV/AIDS were recorded during the period from 2007 to 2017 in the municipality of Ribeirão Preto - SP, 603 of them $(n=603)$ belonging to the category of exposure of men who have sex with men (MSM). Regarding the gender variable, most of the study population consisted of men $70.37 \%(n=1102)$, with 634 cases of white race/color. Regarding schooling, the results showed that $27.5 \%$ $(n=14)$ in 2007 and $56.2 \%(n=90)$ in 2017 had completed middle school. Final Considerations: We conclude that there was an increase in the incidence of HIV / AIDS cases in adults, in the analyzed period.
\end{abstract}

Keywords: Health profile, Young adult, Acquired immunodeficiency syndrome.

\section{RESUMEN}

Objetivo: Analice la incidencia y describa los coeficientes de adultos jóvenes, de 20 a 24 años, con VIH / SIDA, en el período de 2007 a 2017. Métodos: Los datos de notificación se tomaron de la base de datos del Sistema de Información de Agravios y Notificación (SINAN), a través del portal de salud del SUS (DataSUS), que se tabularon en gráficos en el programa Excel, que representan las variables: patrones de incidencia en cada año y de manera comparativa entre años, según el sexo y el grupo de edad, educación y color/raza.

${ }^{1}$ Centro Universitário Barão de Mauá (CBM), Ribeirão Preto - SP. *E-mail: nascimento_igor_@hotmail.com SUBMETIDO EM: 6/2020 | ACEITO EM: 6/2020 PUBLICADO EM: 9/2020 
Resultados: 1124 casos ( $n=1124$ ) de VIH / SIDA se registraron entre 2007 y 2017 en la ciudad de Ribeirão Preto - SP. En la categoría de exposición hombres que tienen sexo con hombres (HSH), mostró 603 casos (n $=603)$. En cuanto a la variable de género, la población estudiada es principalmente hombres, $70.37 \%(n=$ 1102); raza/color blanco, 634 casos $(n=634)$. Con respecto a la educación, en los hombres, los resultados mostraron que en 2007 , el $27.5 \%(n=14)$ había completado la escuela secundaria y en 2017 , el $56.2 \%(n=$ 90). Consideraciones finales: Se concluye que hubo un aumento en la incidencia de casos de VIH / SIDA en adultos, en el período analizado.

Palabras clave: Perfil de salud, Adulto joven, Síndrome de inmunodeficiencia adquirida.

\section{INTRODUÇÃO}

A Síndrome da Imunodeficiência Adquirida (AIDS) é uma doença emergente, grave, causada pelo retrovírus HIV (vírus da imunodeficiência humana) sendo considerado, desde $1981 \mathrm{e}$, atualmente, um dos maiores problemas de saúde pública no Brasil e no mundo (MORESCO EC, et al., 2018; ARAÚJO VLB, et al., 2007).

Historicamente, quando o HIV foi anunciado pelo Center for Desease Control, Atlanta, EUA, em 1981, associava-se a infeção por este vírus, exclusivamente, aos hemofílicos, homens homossexuais, profissionais do sexo e aos usuários de drogas injetáveis (UDI) (BRITO FG, et al., 2014).

No entanto, com o passar dos anos, observou-se modificação no perfil epidemiológico da epidemia de AIDS em todo o mundo, não só para grupos isolados (homossexuais, prostitutas e usuários de drogas injetáveis), a saber: pauperização, feminização e heterossexualização. (SANTOS NJS, et al., 2002).

No mundo, diariamente, mais de 14 mil pessoas são infectadas pelo HIV e, desde o início da epidemia, 20 milhões de pessoas faleceram. Até o ano de 2018, 1,7 milhão de pessoas foram infectadas pelo vírus e até 2010, a doença deixou na orfandade 25 milhões de crianças. Caso não sejam implantadas ações eficazes para combater esse vírus, segundo a projeção da Organização Mundial de Saúde (OMS), 70 milhões de vidas estarão afetadas nos próximos 20 anos. (MORESCO EC, et al., 2018; SILVA RAR, et al., 2013).

Na maioria dos casos, a infecção pelo HIV sem tratamento adequado, leva a uma imunossupressão progressiva. O vírus HIV possui como alvo o sistema imunológico, tornando o organismo inapto a defender futuras infecções oportunistas, acarretando uma desregulação imunitária no indivíduo. Nesse estágio tardio da infecção por HIV se inicia a síndrome da Imunodeficiência Adquirida (AIDS) (MUNIZ FCO, et al., 2018).

A AIDS destrói os linfócitos T CD4+, modificando o material genético dessas células e se replicando em cópias para continuar sua multiplicação e infecção no organismo humano (MUNIZ FCO, et al., 2018; PINHEIRO MC, 2008). As infecções oportunistas em pacientes jovens se manifestam, na maioria dos casos, associadas a múltiplos sintomas, como sarcoma de Kaposi, pneumonias bacterianas, tuberculose, mudanças metabólicas associadas a lipodistrofia, linfaadenopatia localizadas ou generalizadas e inibição geral do sistema imunológico. Apesar de muitas conquistas e avanços, esta síndrome continua desafiando os sistemas de saúde mundialmente, devido sua complexidade clínica e estigmas sociais envolvidos (PEREIRA BPM, et al., 2016).

Segundo estimativas do Programa Conjunto das Nações Unidas sobre HIV/AIDS (UNAIDS) para 2015, 38,8 milhões de pessoas viviam com o HIV no mundo, e destas, 2,1 milhões representavam novas infecções naquele ano. No mesmo ano, cerca de 17 milhões de pessoas se encontrariam em tratamento e teriam ocorrido 1,1 milhão de mortes pela doença. Estima-se que cerca de $50 \%$ das pessoas vivendo com HIV necessitem de tratamento e muitas desconheçam seu status sorológico (KAZANJIAN P, 2017).

A ocorrência de HIV entre os adolescentes apresenta as maiores taxas de novas infecções, sendo a faixa etária dos 15 aos 24 anos responsáveis por quase metade dos novos casos de HIV. Supõe-se que a maioria das infecções aconteceu no período da adolescência, uma vez que a doença pode permanecer assintomática por anos (SANTOS CP, et al., 2010). 
No Brasil, a propagação da infecção pelo HIV, no período entre 2010 e 2018 sofreu transformações significativas no seu perfil epidemiológico, o qual apresentou um aumento de $21 \%$ no número de novos casos em oito anos, dados do Programa Conjunto das Nações Unidas sobre HIV/Aids 2018. Corroborando a isto, dados do Boletim Epidemiológico de 2015 do Ministério da Saúde (MS), nos últimos dez anos, evidenciam um aumentou em $41 \%$ do número de jovens de 20 a 24 anos com HIV (MOREIRA PA, et al., 2019; UNAIDS 2018; BRASIL 2015).

No contexto brasileiro, de 1981 a junho de 2017, já foram notificados no país 882.810 casos de AIDS, dos quais $52,3 \%$ são oriundos da região sudeste. Anualmente, o país tem registrado uma média de 40 mil novos casos de AIDS nos últimos cinco anos. A região sudeste, no período entre 2011 e 2015 apresentou em média 16,8 mil casos ao ano e nos últimos cinco anos (2012 e 2016), 16,3 mil casos ao ano (BRASIL, 2017).

Do ano 2000 a junho de 2017, registrou-se um total de 673.634 casos de AIDS, sendo que 478.940 foram notificados no SINAN (Sistema Nacional de Notificação), 50.399 no SIM (Sistema de Informações de Mortalidade) e 144.295 no SISCEL (Sistema de Controle de Exames Laboratoriais da Rede Nacional de Contagem de Linfócitos CD4+/CD8 e Carga Viral). Porém as regiões Sudeste e Sul apresentam tendência de queda nos últimos dez anos; em 2006, as taxas de detecção foram de 23,5 e 30,2, passando para 17,3 e 25,3 casos/100 mil habitantes em 2016 (BRASIL, 2018).

Atento a esta demanda, desde 1985, o Ministério da Saúde implementou o Programa Nacional de DST/AIDS, objetivando prestar assistência integral às pessoas acometidas por estas infecções, bem como promover ações de promoção à saúde visando diminuir a incidência de tais enfermidades (OLIVEIRA LV, et al., 2017).

Todavia, ainda que reconhecidamente o Brasil tenha alcançado muitos avanços no combate ao HIV/AIDS, percebem-se lacunas no que é preconizado pelas políticas de saúde e assistência prestada às pessoas com HIV/AIDS, bem como baixa resolutividade no tocante a estratégias de prevenção, dado o número significativo de infecção por via sexual, sobretudo em adultos jovens, compreendidos na faixa etária de 20 e 24 anos de idade (TAVARES TRP, et al., 2018).

Em Ribeirão Preto SP, segundo dados da Secretaria Municipal de Saúde a taxa de infecção do HIV entre jovens do sexo masculino entre 20 e 24 anos em 2007 era de 11,8 para cada grupo de 100 mil habitantes e em 2015, a proporção subiu para 79. Entre de jovens de 15 a 19 anos, o número de casos subiu 13,1 em 2007 para 42,2 por 100 mil habitantes em 2015 (RIBEIRÃO PRETO, 2017). Em 2017, Ribeirão Preto - SP era o 74- município brasileiro em números de casos de AIDS, no Ranking dos 100 municípios com mais de 100 mil habitantes de 2012 a 2016, com 23,6 de taxa de detecção (BRASIL, 2017).

Em relação aos coeficientes de mortalidade, do total de óbitos por AIDS registrados no Brasil no período entre 1980 até 31 de dezembro de 2016, foram notificados no Brasil $(n=316.088)$ óbitos tendo a HIV/AIDS como causa básica. A maior proporção destes óbitos ocorreu na região Sudeste (59,6\%), seguida das regiões Sul $(17,6 \%)$, Nordeste $(13,0 \%)$, Centro Oeste $(5,1 \%)$ e Norte $(4,7 \%)$. (BRASIL, 2016). Além disso, entre indivíduos do sexo masculino, também se verificou uma tendência de aumento entre aqueles compreendidos na faixa etária de 20 a 24 anos, passando de 2,7 óbitos por 100 mil habitantes em 2006 para 3,6 óbitos por 100 mil habitantes em 2016. (RIBEIRÃO PRETO, 2017).

O panorama em que se encontra o HIV/AIDS na atualidade fundamentou o objetivo deste trabalho para descrever o perfil epidemiológico de adultos jovens com HIV/AIDS no município de Ribeirão Preto SP, no período de 2007 a 2017. Em suma, os resultados obtidos podem disponibilizar uma valiosa fonte de informações para o planejamento de ações voltadas à prevenção e controle do HIV e da AIDS nesta faixa etária, como também fornecer uma base de consulta para outros estudos de avaliação da eficácia das estratégias implantadas.

\section{MÉTODOS}

Trata-se de um estudo ecológico descritivo, retrospectivo, com abordagem quantitativa, a fim de analisar o perfil demográfico da AIDS em adultos jovens com HIV/AIDS, em Ribeirão Preto SP, no período de 2007 a 2017. Os dados de notificação foram coletados da base de dados do Sistema de Informações de Agravos de 
Notificação (SINAN), via Portal de Saúde do SUS (DataSUS), disponibilizados pela Divisão de Epidemiologia da Secretaria Municipal de Saúde de Ribeirão Preto SP. A população do estudo foi constituída de todos os dados de notificação dos indivíduos notificados com AIDS residentes em Ribeirão Preto - SP no período de 2007 a 2017.

Por questões ético-legais o estudo foi encaminhado para avaliação e parecer do Comitê de Ética em Pesquisa do Centro Universitário Barão de Mauá via Plataforma Brasil, sendo aprovado conforme parecer 3.961.291, sob o registro de $n^{\circ} 29650719.1 .0000 .5378$, cumprindo os preceitos éticos elencados na resolução 466/2012 do Conselho Nacional de Saúde (CNS).

Por não fazer uso de amostras biológicas, sendo utilizados somente dados coletos em fichas epidemiológicas, foi dispensado o uso do Termo de Consentimento Livre e Esclarecido. As informações coletadas foram organizadas em planilhas utilizando o programa Microsoft Excel $2016 \mathrm{com}$ as seguintes variáveis: Prevalência dos sexos, faixa etária, categoria de exposição, escolaridade e raça/cor.

Foram observados os padrões de incidência em cada ano e de maneira comparativa entre os anos, segundo sexo e faixa etária. Mediante a análise de tais dados, foram construídos os resultados, discussão e conclusão.

\section{RESULTADOS E DISCUSSÃO}

Para melhor definição dos dados da referida pesquisa e compreensão dos resultados obtidos, a distribuição de frequência dos casos notificados de HIV/AIDS foram agrupados em forma de tabela, correspondendo à categoria de exposição, em jovens de 20 a 24 anos, de maneira comparativa entre os anos (Tabela 1).

Tabela 1 - Distribuição de frequências dos casos notificados de HIV/AIDS, em jovens, de 20 a 24 anos, no município de Ribeirão Preto - SP.

\begin{tabular}{lcccccccccc}
\hline & \multicolumn{1}{c}{ Categoria de exposição } \\
\hline & \multicolumn{1}{c}{ HSH } & \multicolumn{1}{c}{ Heterossexual } & \multicolumn{3}{c}{ UDI } & \multicolumn{3}{c}{ Ignorado } & \multicolumn{2}{c}{ Total } \\
\hline Ano de diagnóstico & $\mathbf{N}$ & $\%$ & $\mathbf{N}$ & $\%$ & $\mathbf{N}$ & $\%$ & $\mathbf{N}$ & $\%$ & \multicolumn{1}{c}{$\mathbf{~}$} & $\%$ \\
\hline 2007 & 14 & 27,0 & 24 & 47,0 & 4 & 8,0 & 9 & 18,0 & 51 & 100,0 \\
2008 & 25 & 37,0 & 32 & 47,0 & 3 & 4,0 & 8 & 12,0 & 68 & 100,0 \\
2009 & 31 & 47,0 & 22 & 34,0 & 2 & 3,0 & 10 & 15,0 & 133 & 100,0 \\
2010 & 30 & 51,0 & 15 & 25,0 & 3 & 5,0 & 10 & 17,0 & 58 & 100,0 \\
2011 & 29 & 43,0 & 23 & 33,0 & 2 & 3,0 & 13 & 19,0 & 6 & 100,0 \\
2012 & 53 & 50,0 & 32 & 34,0 & 5 & 5,0 & 16 & 15,0 & 106 & 100,0 \\
2013 & 64 & 54,0 & 33 & 30,0 & 6 & 5,0 & 15 & 13,0 & 118 & 100,0 \\
2014 & 79 & 59,0 & 36 & 27,0 & 5 & 4,0 & 14 & 10,0 & 134 & 100,0 \\
2015 & 93 & 65,0 & 28 & 20,0 & 3 & 2,0 & 18 & 13,0 & 142 & 100,0 \\
2016 & 81 & 61,0 & 23 & 17,0 & 5 & 4,0 & 24 & 18,0 & 133 & 100,0 \\
2017 & 104 & 65,0 & 31 & 19,0 & 2 & 1,0 & 23 & 14,0 & 160 & 100,0 \\
\hline Total & 603 & 559,0 & 299 & 333,0 & 38 & 44,0 & 160 & 164,0 & 1124 & 100,0 \\
\hline
\end{tabular}

Legenda: HSH: Homens que fazem sexo com homens, UDI: Uso de drogas injetáveis, N: Frequência absoluta, \%: Frequência relativa representada em porcentagem.

Fonte: Alves IN, et al., 2020. Baseado em Sinan NET 2017/ SIM/ DVE RP/ DEVISA/ IBGE/ PRMP/ MS.

Segundo os dados do Sistema de Informação de Agravos de Notificação (SINAN) foram registrados 1124 casos ( $n=1124$ ) de HIV/AIDS no período de 2007 a 2017 no município de Ribeirão Preto - SP. No qual, houve um aumento $213,72 \%$ de número de infectados notificados nesses últimos dez anos. A categoria que apresentou maior exposição foi a de homens que fazem sexo com homens (HSH), a qual evidenciou uma prevalência de 603 casos ( $n=603$ ) no período de 2007 a 2017 e um aumento de 642,85\%, seguido por heterossexual, UDI e ignorado. 
Tabela 2 - Taxa de detecção de HIV/AIDS segundo faixa etária e sexo, Ribeirão Preto - SP, 2007 e 2017.

\begin{tabular}{|c|c|c|c|}
\hline \multicolumn{4}{|c|}{ Homens } \\
\hline Faixa etária & Casos/100 mil habitantes (2007) & Casos/100 mil habitantes (2017) & Variação \\
\hline 13- 14 & 0,0 & 0,0 & $0,00 \%$ \\
\hline 15- 19 & 13,2 & 29,6 & $124,24 \%$ \\
\hline $20-24$ & 11,9 & 133,3 & $1020,16 \%$ \\
\hline 25- 29 & 42,9 & 142,4 & $231,93 \%$ \\
\hline 30- 39 & 46,9 & 77,5 & $65,24 \%$ \\
\hline 40- 49 & 26,5 & 36,1 & $36,22 \%$ \\
\hline $50-59$ & 10,8 & 2,4 & $-77,70 \%$ \\
\hline $60-69$ & 6,3 & 0,0 & $-100,00 \%$ \\
\hline 70 ou mais & 0,0 & 0,0 & $0,00 \%$ \\
\hline \multicolumn{4}{|c|}{ Mulheres } \\
\hline Faixa etária & Casos/100 mil habitantes (2007) & Casos/100 mil habitantes (2017) & Variação \\
\hline 13- 14 & 0,0 & 0,0 & $0,00 \%$ \\
\hline 15- 19 & 13,2 & 11,3 & $-14,39 \%$ \\
\hline $20-24$ & 38,1 & 21,9 & $-42,51 \%$ \\
\hline $25-29$ & 51,9 & 8,9 & $-82,85 \%$ \\
\hline $30-39$ & 26,2 & 17,7 & $-32,44 \%$ \\
\hline 40- 49 & 23,6 & 12,2 & $-48,30 \%$ \\
\hline $50-59$ & 12,1 & 8,6 & $-28,92 \%$ \\
\hline $60-69$ & 0,0 & 9,8 & $980,00 \%$ \\
\hline 70 ou mais & 0,0 & 0,0 & $0,00 \%$ \\
\hline
\end{tabular}

Fonte: Alves IN, et al., 2020. Baseado em Sinan NET 2017/ SIM/ DVE RP/ DEVISA/ IBGE/ PRMP/MS.

Diferente dos homens, as mulheres apresentaram queda nos casos na maioria das faixas etárias no mesmo período. Com médias menores em função da queda nos casos relacionados ao sexo feminino, houve elevação em faixas etárias, do período de 2007 a 2017, apenas entre 60 a 69 anos (980\%), com 9,8 casos por 100 mil pessoas (Tabela 2). De acordo com o estudo, a taxa de infecção do HIV entre jovens do sexo masculino entre 20 e 24 anos em 2007 era de 11,8 para cada grupo de 100 mil habitantes. Em 2017, a proporção subiu para 133,1020,16\% de variação percentual de aumento. Entre jovens de 15 a 19 anos, a incidência subiu 124,24\%, partindo de 13,2 casos para 29,6 por 100 mil habitantes. Nas demais faixas etárias, houve um aumento de casos, apenas 50 a 59 e 60 a 69, que obtiveram decréscimo e 13 a 14 que não houve variação percentual.

A AIDS tornou-se uma pandemia, um ícone de grandes questões que afligem o planeta, como direitos humanos, qualidade de vida, políticas de medicamentos e propriedade industrial. Constitui-se, por conseguinte, um fenômeno, cuja forma de ocorrência, nas diferentes partes do mundo, configura-se como epidemias regionais com características e determinantes próprias (PINTO ACS, et al., 2007).

Sabe-se que o comportamento da HIV/AIDS difere de acordo com os determinantes sócios demográficos dispostos em cada cenário do país e do mundo (LAZARINI FM, 2011). O Brasil vive um contexto de aumento da prevalência de HIV entre os adultos jovens, e a epidemia de AIDS é concentrada em alguns subgrupos populacionais principalmente entre HSH; prevalências elevadas de HIV em alguns grupos populacionais em contextos de vulnerabilidade (HSH, trabalhadoras sexuais - TS, UDI, travestis e transexuais - TT) chamados hoje de populações-chave, e tendência de redução do uso regular do preservativo (BRASIL, 2013; SZWARCWALD CL, et al., 2011; WORLD HEALTH ORGANIZATION, 2014; CORDEIRO AM, et al.,2007).

Observa-se perante o estudo um número expressivo na proporção de $\mathrm{HSH}$, o que coaduna com os dados do Boletim Epidemiológico, onde há uma tendência de aumento na proporção dos casos de homens que fazem sexo com homens nos últimos oito anos, passando de 27,0\% em 2007 para 65\% em 2015 (BRASIL, 2016). Essa alta incidência de casos notificados entre os mais novos se deve a uma série de situações de risco adotadas, sobretudo por questões culturais como o abandono do uso do preservativo nas relações sexuais ou após o início de um relacionamento (DOURADO I, et al., 2015; BRASIL 2016). 
No Brasil, existe, desde 2010, a profilaxia pós-exposição (PEP), adotada após um comportamento de risco ou por profissionais de saúde que possam ter se infectado ao tratar pacientes. Porém, apesar dos tratamentos com medicamentos e avanços da ciência que garantem a qualidade de vida aos pacientes, o HIV/AIDS ainda é uma doença que não tem cura e a melhor maneira é a prevenção, como a utilização de preservativos (MINISTÉRIO DA SAÚDE, 2018).

O uso do preservativo, masculino ou feminino, constitui-se no único método que impede a propagação do HIV/AIDS por via sexual, além das demais infecções sexualmente transmitidas (ISTs). Apesar de extensas campanhas, a taxa de uso do preservativo está aquém do esperado (TAMAYO A, et al., 2001).

O uso do preservativo é dever do portador do HIV/AIDS, em virtude da ampla transmissão mediante a relação sexual, o que reflete a consciência e a responsabilidade da pessoa acometida e seu parceiro (FELISMINO HP, et al., 2008).

Tais dados apresentados nesse presente estudo refletem a importância do uso de preservativo durante todas as relações sexuais e também da orientação de seu uso pelos profissionais de saúde a qualquer pessoa, seja ela adolescente ou adulta (THAMARA SC, LILIANE CCR, 2011). Assim, faz-se necessário reconhecer a importância do preservativo masculino e feminino no âmbito da política de prevenção do HIV/AIDS, no Brasil e no mundo (DOURADO I et al, 2015).

Tabela 3 - Prevalência de casos de HIV/AIDS segundo sexo por ano de diagnóstico, no município de Ribeirão Preto SP, 2007 a 2017.

\begin{tabular}{lllllllllllllll}
\hline & 2007 & 2008 & 2009 & 2010 & 2011 & 2012 & 2013 & 2014 & 2015 & 2016 & 2017 & Total \\
\hline Variáveis & $\mathbf{N}$ & $\mathbf{N}$ & $\mathbf{N}$ & $\mathbf{N}$ & $\mathbf{N}$ & $\mathbf{N}$ & $\mathbf{N}$ & $\mathbf{N}$ & $\mathbf{N}$ & $\mathbf{N}$ & $\mathbf{N}$ & $\mathbf{N}$ \\
\hline Sexo & & & & & & & & & & & & & \\
\hline Masculino & 51 & 68 & 65 & 59 & 67 & 106 & 116 & 135 & 142 & 133 & 160 & 1102 \\
Feminino & 53 & 48 & 47 & 41 & 33 & 40 & 39 & 40 & 46 & 42 & 35 & 464 \\
\hline
\end{tabular}

Legenda: N: Frequência absoluta, \%: Frequência relativa representada em porcentagem.

Fonte: Alves IN, et al., 2020. Baseado em Sinan NET 2017/ SIM/ DVE RP/ DEVISA/ IBGE/ PRMP/MS.

Em relação a variável de gênero entre homens e mulheres, a população estudada constitui-se em sua maioria de homens $70,37 \%(n=1102)$, enquanto $29,62 \%(n=464)$ são mulheres (Tabela 3). Atualmente a epidemiologia do HIV/AIDS é caracterizada pelo aumento de casos na faixa etária jovem, populações de baixa renda e baixa escolaridade (BRITO FG, et al., 2014; ABREU SR, et al., 2016). Diante dos dados elucidados neste estudo, o perfil sócio demográfico dos jovens que vivem com HIV/AIDS na cidade de Ribeirão Preto SP acompanha as características da epidemia no Brasil.

Em relação ao número de casos novos infectados pela AIDS, do ano 2000 a junho de 2017, registrou-se um total de 673.634 indivíduos, sendo a razão de sexos para o ano de 2016, desconsiderando casos de HIV em gestantes, de 2,5 homens para cada mulher infectada (BRASIL, 2017).

De acordo com o Boletim Epidemiológico do Ministério da Saúde (2016), o número de homens diagnosticados com HIV/AIDS ainda é superior ao de mulheres. (BRASIL, 2016). No entanto, a literatura sinaliza o aumento do número de mulheres infectadas pelo HIV/AIDS em todo o mundo. Brito et al, (2014) e Abreu et al, (2016) relevaram em seus estudos, realizados com mulheres casadas e soropositivas, que a união prolongada com o mesmo parceiro, é equivocadamente considerada fator de proteção, o que possivelmente tem contribuído para feminização da epidemia (BRITO FG, et al., 2014; ABREU SR, et al., 2016).

Uma pesquisa realizada pelo MS (2008) revelou alguns fatores que contribuem para o aumento da vulnerabilidade de adolescentes e jovens ao HIV. Entre eles estão à dificuldade no acesso à informação; 0 pouco reconhecimento de direitos sexuais e reprodutivos de adolescentes e jovens; os estigmas e preconceitos (de gênero, identidade de gênero, raça/etnia, orientação sexual, entre outros); pouco diálogo com a família, especialmente sobre sexualidade; e a baixa frequência de adolescentes e jovens nos serviços de saúde (BRASIL, 2012; MOREIRA PA, et al., 2019). 
Outros fatores que propiciam o contágio de HIV/ AIDS são a baixa idade das primeiras relações sexuais, a falta de informação referente à realização do ato sexual, a variabilidade de parceiros, a utilização de álcool, o uso de drogas ilícitas e o não uso de preservativo (UNAIDS, 2018).

Estima-se que 11,8 milhões de jovens de 15 a 24 anos vivem com HIV/AIDS hoje em dia em todo o mundo. A cada dia, cerca de 6 mil jovens dessa faixa etária se infectam com o HIV (MOREIRA PA et al,2019; BRASIL, 2006). Das 4.500 novas infecções por HIV, no mundo, em adultos no ano de 2016 , 35\% ocorreu entre jovens de 15 a 24 anos, e anualmente uma em cada dez pessoas sexualmente ativas adquire uma IST (UNAIDS, 2018). São necessárias intervenções mais efetivas, não apenas no campo da informação e da prevenção por meio de campanhas públicas, mas também incorporando parcerias com outras instituições, como escolas e programas de saúde da família (BRASIL, 2011)

A educação em saúde com orientações familiares, escolares e investimentos públicos é importante para prevenção do HIV em jovens (BRASIL, 2012). Nesse sentido, é possível afirmar que alguns fatores contribuam para mudança do padrão de comportamento sexual, como, por exemplo, acesso a vários tipos de materiais informativos e educativos nas escolas sobre atividade sexual e prevenção das IST's, bem como o acesso à internet, distribuição de preservativos em diferentes ambientes, o diálogo com os pais sobre relação sexual e o acesso ao sistema de saúde (BRASIL, 2011).

Tabela 4 - Distribuição dos casos notificados de HIV/AIDS, segundo raça/cor, por ano de diagnóstico, em residentes do sexo masculino em Ribeirão Preto SP, 2007 a 2017.

\begin{tabular}{lcccccccccccccc}
\hline & \multicolumn{10}{c}{ Raça/Cor } \\
\hline & \multicolumn{1}{c}{ Branca } & \multicolumn{1}{c}{ Preta } & \multicolumn{1}{c}{ Parda } & \multicolumn{1}{c}{ Amarela } & \multicolumn{1}{c}{ Indígena } & \multicolumn{2}{c}{ S/inf } & \multicolumn{2}{c}{ Total } \\
\hline Ano de diagnóstico & $\mathbf{N}$ & $\%$ & $\mathbf{N}$ & $\%$ & $\mathbf{N}$ & $\%$ & $\mathbf{N}$ & $\%$ & $\mathbf{N}$ & $\%$ & $\mathbf{N}$ & $\%$ & $\mathbf{N}$ & $\%$ \\
\hline 2007 & 30 & 58,8 & 3 & 5,9 & 8 & 15,7 & 0 & 0,0 & 0 & 0,0 & 10 & 19,6 & 51 & 100,0 \\
2008 & 36 & 52,9 & 5 & 7,4 & 14 & 20,6 & 0 & 0,0 & 0 & 0,0 & 13 & 19,1 & 68 & 100,0 \\
2009 & 29 & 44,6 & 9 & 13,8 & 21 & 32,3 & 0 & 0,0 & 0 & 0,0 & 6 & 9,2 & 65 & 100,0 \\
2010 & 33 & 55,9 & 4 & 6,8 & 14 & 23,7 & 0 & 0,0 & 0 & 0,0 & 8 & 13,6 & 59 & 100,0 \\
2011 & 36 & 53,7 & 8 & 11,9 & 11 & 16,4 & 0 & 0,0 & 0 & 0,0 & 12 & 17,9 & 67 & 100,0 \\
2012 & 64 & 60,4 & 12 & 11,3 & 19 & 17,9 & 0 & 0,0 & 0 & 0,0 & 11 & 10,4 & 106 & 100,0 \\
2013 & 71 & 60,2 & 6 & 5,1 & 25 & 21,2 & 1 & 0,8 & 0 & 0,0 & 15 & 12,7 & 118 & 100,0 \\
2014 & 78 & 57,8 & 12 & 8,9 & 27 & 20,0 & 0 & 0,0 & 0 & 0,0 & 18 & 13,3 & 135 & 100,0 \\
2015 & 86 & 60,6 & 7 & 4,9 & 21 & 14,8 & 1 & 0,7 & 0 & 0,0 & 27 & 19,0 & 142 & 100,0 \\
2016 & 77 & 57,9 & 10 & 7,5 & 28 & 21,1 & 1 & 0,8 & 0 & 0,0 & 17 & 12,8 & 133 & 100,0 \\
2017 & 94 & 58,8 & 8 & 5,0 & 26 & 16,3 & 2 & 1,3 & 0 & 0,0 & 30 & 18,8 & 160 & 100,0 \\
\hline
\end{tabular}

Legenda: N: Frequência absoluta, \%: Frequência relativa representada em porcentagem. Fonte: Alves IN, et al., 2020. Baseado em Sinan NET 2017/ SIM/ DVE RP/ DEVISA/ IBGE/ PRMP/ MS.

Em relação à distribuição de casos segundo raça/cor destaca se a raça branca, na qual observa se maior número de casos em todo o período pesquisado. A cor branca totalizou 634 casos ( $n=634)$, e em seguida, indivíduos de raça parda, totalizaram 214 ( $n=214$ ) casos nesses dez anos em estudo (Tabela 4).

No que concerne à distribuição racial dos indivíduos analisados, constata se um alto nível de soropositivos autodeclarados brancos, seguidos de pardos (Tabela 4), dados que contrapõem com os dos registrados no Sistema de Informação de Agravos de Notificação (SINAN), o qual identifica que em âmbito nacional, 54,8\% das pessoas com HIV/AIDS são pretos e pardos e 44,0\% são brancos (BRASIL, 2016).

Porém, esses dados apresentados no presente estudo corroboram com o Ministério da Saúde (2013), o qual observa se, $48,4 \%$ dos casos notificados entre os homens se auto declararam de raça/cor branca e $41,3 \%$ de parda (BRASIL MS, 2013). 
Tabela 5 - Proporção dos casos de HIV/AIDS notificados, segundo escolaridade e ano de diagnóstico, em residentes do sexo masculino em Ribeirão Preto SP, 2007 a 2017.

\begin{tabular}{|c|c|c|c|c|c|c|c|c|c|c|c|c|}
\hline \multicolumn{13}{|c|}{ Escolaridade } \\
\hline & \multicolumn{2}{|c|}{ Analfabeto } & \multicolumn{2}{|c|}{ Ignorado } & \multicolumn{2}{|c|}{ En Fund I } & \multicolumn{2}{|c|}{ En Fund C } & \multicolumn{2}{|c|}{ En Méd C } & \multicolumn{2}{|c|}{ Total } \\
\hline Ano de diagnóstico & $\mathbf{N}$ & $\%$ & $\mathbf{N}$ & $\%$ & $\mathbf{N}$ & $\%$ & $\mathbf{N}$ & $\%$ & $\mathbf{N}$ & $\%$ & $\mathbf{N}$ & $\%$ \\
\hline 2007 & 0 & 0,00 & 19 & 37,25 & 6 & 11,70 & 12 & 23,52 & 14 & 27,50 & 51 & 100,0 \\
\hline 2008 & 0 & 0,00 & 20 & 29,50 & 22 & 32,35 & 12 & 17,64 & 14 & 20,58 & 68 & 100,0 \\
\hline 2009 & 0 & 0,00 & 18 & 27,70 & 14 & 21,50 & 15 & 23,10 & 18 & 27,70 & 65 & 100,0 \\
\hline 2010 & 3 & 5,08 & 21 & 35,60 & 10 & 16,95 & 10 & 16,95 & 15 & 25,42 & 59 & 100,0 \\
\hline 2011 & 1 & 1,50 & 22 & 32,85 & 14 & 20,90 & 7 & 10,45 & 23 & 34,30 & 67 & 100,0 \\
\hline 2012 & 1 & 0,95 & 30 & 28,30 & 16 & 15,10 & 14 & 13,20 & 45 & 42,45 & 106 & 100,0 \\
\hline 2013 & 1 & 0,84 & 32 & 27,11 & 17 & 14,40 & 18 & 15,25 & 50 & 42,40 & 118 & 100,0 \\
\hline 2014 & 0 & 0,00 & 43 & 31,85 & 13 & 9,63 & 14 & 10,37 & 65 & 48,15 & 135 & 100,0 \\
\hline 2015 & 0 & 0,00 & 47 & 33,10 & 15 & 10,56 & 10 & 7,04 & 70 & 49,30 & 142 & 100,0 \\
\hline 2016 & 2 & 1,48 & 46 & 34,00 & 11 & 8,20 & 12 & 8,90 & 64 & 47,40 & 135 & 100,0 \\
\hline 2017 & 0 & 0,00 & 46 & 28,75 & 11 & 6,87 & 13 & 8,12 & 90 & 56,25 & 160 & 100,0 \\
\hline
\end{tabular}

Legenda: N: Frequência absoluta, \%: Frequência relativa representada em porcentagem. En Fund I: Ensino fundamental incompleto, En Fund C: Ensino fundamental completo, En Méd C: Ensino médio completo.

Fonte: Alves IN, et al., 2020. Baseado em Sinan NET 2017/ SIM/ DVE RP/ DEVISA/ IBGE/ PRMP/ MS.

No que diz respeito à escolaridade, em homens, observa se um crescimento dos anos escolares durante os períodos. Os resultados demonstraram que em $2007,27,5 \%(n=14)$ apresentavam ensino médio completo e em 2017, 56,2\% ( $n=90)$

No entanto, a variável registrou 2007 a 2017, 344 casos $(n=344)$ com a categoria "ignorado", à frente de: analfabeto, ensino fundamental incompleto e completo. Tal fato indica a fragilidade de análise e interpretações de dados e a necessidade de uma melhora na qualidade dos registros das informações.

Sabe se que a pauperização é representada pela baixa escolaridade, de forma que indivíduos com menor grau escolar pode implicar em prejuízos à adesão ao tratamento e também interferindo na compreensão da doença (ABREU, 2016). A baixa escolaridade se comporta como variável representante da má distribuição de renda e da pobreza, das precárias condições de vida, trabalho, transporte, habitação e acesso a serviços de saúde, evidenciando um quadro persistente de desigualdades. (DAMIANA B, 2015)

Por falta de dados pertinentes, as variáveis: saúde dos parceiros sexuais e orientação sexual, não foram incluídas no presente estudo, embora sejam avaliadas na literatura consultada (LANNUZYA VO, et al., 2017).

O desconhecimento acerca da situação de saúde dos parceiros da população estudada torna-se preocupante, e pode demonstrar lacunas na assistência à saúde - a qual deve atender não só a pessoa com HIV/AIDS, mas também seus familiares - bem como pode resultar dos estigmas e preconceitos que permeiam este quadro, e que talvez favoreça a quebra de vínculos entre a população em estudo e seus parceiros (BORTOLOTTI LR, et al., 2014).

\section{CONSIDERAÇÕES FINAIS}

Pela observação dos aspectos analisados pôde-se concluir que o perfil de adultos jovens (20 a 24 anos) com HIV/AIDS em uma cidade do interior paulista de 2007 a 2017 caracteriza - se predominantemente de homens, cor branca e ensino médio completo. Foi observado um aumento na incidência dos casos de AIDS em adultos, no período de 2007 a 2017. Em 2017, Ribeirão Preto SP era o 74 município brasileiro em números de casos de AIDS. Sendo assim, acredita-se que os resultados apresentados nesse estudo podem nortear melhorias nas ações de educação em saúde, com ênfase na prevenção da transmissão do HIV. Além de possibilitar a tomada de decisão dos gestores locais e dos profissionais em saúde na melhoria da assistência aos jovens e adultos, contribuindo assim para mudanças no quadro epidemiológico da AIDS. 


\section{REFERÊNCIAS}

1. ABREU SR, et al. Estudo epidemiológico de pacientes com infecção pelo vírus da imunodeficiência humana/síndrome da imunodeficiência adquirida (hiv/aids), Caxias-MA. Revista Interdisciplinar, Maranhão, v. 9, n. 4, p. 132-141, out. nov. dez. 2016.

2. ARAUJO VLB, et al. Características da Aids na terceira idade em um hospital de referência do Estado do Ceará, Brasil. Rev. bras. epidemiol., São Paulo, v. 10, n. 4, p. 544-554, Dec. 2007.

3. BORTOLOTTI LR, et al. O significado do viver com o HIV/AIDS na adolescência: estudo descritivo. OBJN, v. 13, n.4, p.53748, 2014.

4. BRASIL. Boletim Epidemiológico HIV/AIDS. Departamento de Vigilância, Prevenção e Controle das IST, HIV/Aids e Hepatites Virais. Brasília: Ministério da Saúde, v. XX, n. 8, 2017. Disponível em: <aids.gov.br/pt-br/pub/2017/boletimepidemiologico-hivaids-2017>.

5. BRASIL. Boletim Epidemiológico HIV/AIDS. Departamento de Vigilância, Prevenção e Controle das IST, HIV/Aids e Hepatites Virais. Brasília: Ministério da Saúde, 2016.

6. BRASIL. Ministério da Saúde (MS). Secretaria de Atenção a Saúde. Programa Nacional de DST/Aids. Boletim Epidemiológico Aids e DST. Brasília: MS; 2012.

7. BRASIL. Ministério da Saúde (MS). Secretaria de Vigilância em Saúde. Departamento de DST, Aids e Hepatites Virais. Recomendações para a Atenção Integral a Adolescentes e Jovens Vivendo com HIV/Aids. 1a Edição. Brasília: MS; 2013.

8. BRASIL. Ministério da Saúde. Manual de rotinas para assistência a adolescentes vivendo com HIV/Aids. Brasília, DF: Ministério da Saúde, 2006. 168 p.

9. BRASIL. Ministério da Saúde. Secretaria de Vigilância em Saúde. Departamento de Vigilância, Prevenção e Controle das Infecções Sexualmente Transmissíveis, do HIV/Aids e das Hepatites Virais. Boletim Epidemiológico HIV/AIDS 2018, 2018; 49(53): 01-72.

10. BRASIL. Ministério da Saúde. Secretaria de Vigilância em Saúde. Departamento de DST, AIDS e Hepatites Virais Boletim Epidemiológico Aids e DST. Brasília: Ministério da Saúde; 2013.

11. BRASIL. Ministério da Saúde. Secretaria de Vigilância Sanitária. Programa Nacional de Controle e Prevenção de DST/AIDS. Boletim Epidemiológico AIDS e DST. Brasília, 2011.

12. BRITO FB, et al. Perfil Epidemiológico de Portadores do Vírus da Imunodeficiência Humana e Síndrome da Imunodeficiência Adquirida no estado de Sergipe, 2007-2012. Interfaces Científicas - Saúde e Ambiente, Aracajú, v. 2, n. 2, p.59-72, fev. 2014.

13. BRITO FG, et al. Perfil epidemiológico de portadores do vírus da imunodeficiência humana e síndrome da imunodeficiência adquirida no estado de Sergipe, 2007- 2012. Interfaces Científicas, Aracaju, v.2, n.2, p. 59 - 71, fev., 2014.

14. CORDEIRO AM, et al. Revisão sistemática: uma revisão narrativa. Rev Col Bras Cir 2007; 34: 428-31.

15. DAMIANA B. Fatores associados à tuberculose/hiv no Brasil: Uma análise de dados secundários com ênfase nas variáveis raça/cor, DF. Dissertação (Pós Graduação em Saúde Coletiva) - Faculdade de Ciências da Saúde. Universidade de Brasília, Brasília, 2015.

16. DOURADO I, et al. Revisitando o uso do preservativo no Brasil. Rev. bras. epidemiol., São Paulo, v. 18, supl. 1, p. 63-88, set. 2015.

17. FELISMINO HP, et al. Direitos e deveres de pessoas vivendo com HIV/AIDS no âmbito da saúde: um enfoque bioético. Rev Eletr Enferm. 2008; 10(1):87-99.

18. KAZANJIAN P. UNAIDS 90-90-90 Campaign to End the AIDS Epidemic in Historic Perspective. The Milbank Quarterly, vol. 95, no. 2, p. 408-439, 2017.

19. LANNUZYA VO, et al. Perfil epidemiológico de adultos jovens com HIV/AIDS em Natal, RN: estudo descritivo. Anais II CONBRACIS V. 1, 2017, ISSN 2525-6696.

20. LAZARINI FM. Tendência e Características da Epidemia de Aids em um Município de Grande Porte do Sul do Brasil: 1986 a 2008. Londrina, 2011. Dissertação (Mestrado em Saúde Coletiva) - Universidade Estadual de Londrina, Paraná.

21. MINISTÉRIO DA SAÚDE. Secretaria de Vigilância em Saúde. Departamento de Departamento de Vigilância, Prevenção e Controle das Infecções Sexualmente Transmissíveis, do HIV/Aids e das Hepatites Virais. Protocolo Clínico e Diretrizes Terapêuticas para Profilaxia Pós-exposição (pep) de Risco à Infecção pelo HIV, IST e Hepatites Virais. Brasília. 2018.

22. MOREIRA PA, et al. Vulnerability to HIV/AIDS in adolescents of a public school inside Sergipe. Rev Fun Care Online. 2019 jul/set; 11(4):868-872.

23. MORESCO EC, et al. ASPECTOS GERAIS SOBRE A SÍNDROME DA IMUNODEFICIÊNCIA ADQUIRIDA (AIDS). Anuário Pesquisa e Extensão Unoesc Videira, v. 3, p. e19912, 27 nov. 2018.

24. MUNIZ FCO, et al. Pacientes críticos com hiv/aids: fatores associados às complicações. Dissertação (Monografia no Curso de Pós-graduação em Terapia Intensiva e Alta Complexidade). Escola Bahiana de Medicina e Saúde Pública, Salvador, 2018.

25. OLIVEIRA LV, et al. Perfil Epidemiológico de Adultos Jovens com HIV/Aids em Natal, RN: Estudo Descritivo. II Congresso Brasileiro de Ciências da Saúde, Campina Grande, V. 1, 2017, ISSN 2525-6696, jun. 2017.

26. PEREIRA BPM, et al. Estudo epidemiológico de pacientes com infecção pelo vírus da imunodeficiência humana/síndrome da imunodeficiência adquirida (HIV/aids), Caxias-MA. Revista Interdisciplinar. 2016;9(4):132-41.

27. PINHEIRO MC. Níveis de TNF-A e seu polimorfismo genético em pacientes hiv positivos, portadores de lipodistrofia e em uso de terapia antirretroviral, PA. Dissertação (Mestrado em Doenças Tropicais) - Instituto de Ciências da Saúde. Universidade Federal do Pará, Belém, 2008.

28. PINTO ACS, et al. Compreensão da pandemia de AIDS nos últimos 25 anos. DST: j bras doenças sex transm., v. 19, n. 1, p. 45-50, 2007

29. RIBEIRÃO PRETO. Dados Epidemiológicos HIV/AIDS. Secretaria da Saúde de Ribeirão Preto - Divisão Vigilância Epidemiológica, 2017.

30. SANTOS CP, et al. Vivência das adolescentes e jovens com HIV: um estudo fenomenológico. Adolesc Saude. 2010;7(1):4044. 
31. SANTOS NJS, et al. A aids no Estado de São Paulo: as mudanças no perfil da epidemia e perspectivas da vigilância epidemiológica. Revista Brasileira de Epidemiologia, vol. 5, no. 3, p. 286-310, 2002.

32. SILVA RAR, et al. A Epidemia da Aids no Brasil: Análise do Perfil Atual. Português/Inglês Rev enferm UFPE online, Recife, 7(10):6039-8, out., 2013.

33. SZWARCWALD CL, et al. HIV-related risky practices among Brazilian young men, 2007. Cad Saude Publica $2011 ; 27$ Suppl 1: S19-26.

34. TAMAYO A, et al. Prioridades axiológicas e uso de preservativo. psicologia: Reflex Crít. 2001; 14(1):167-75.

35. TAVARES TRP, et al. "A gente vive em cima da corda bamba": experiência de profissionais da saúde que trabalham com o HIV/aids em uma área remota do Nordeste brasileiro. Cad. Saúde Pública, Rio de Janeiro, v. 34, n. 11, e00063618, 2018.

36. THAMARA SC, LILIANE CCR. Perfil epidemiológico dos pacientes com HIV/AIDS no alto vale do Jequitinhonha, 1995-2008. Rev Med Minas Gerais 2011; 21(1): 14-18.

37. UNAIDS. Estatísticas. Resumo Global da Epidemia de Aids. Acesso em 15 abr. 2018. Disponível em: https://unaids.org.br/estatisticas/.

38. WORLD HEALTH ORGANIZATION. Consolidated guidelines on HIV prevention, diagnosis, treatment and care for key populations. Geneva: World Health Organization; 2014. 